\title{
Pathological assessment of pericolonic tumor deposits in advanced colonic carcinoma: relevance to prognosis and tumor staging
}

\author{
Giacomo Puppa ${ }^{1}$, Patrick Maisonneuve ${ }^{2}$, Angelica Sonzogni ${ }^{3}$, Michele Masullo ${ }^{3}$, \\ Paola Capelli $^{4}$, Marco Chilosi ${ }^{4}$, Fabio Menestrina ${ }^{4}$, Giuseppe Viale ${ }^{3,5}$ and Giuseppe Pelosi ${ }^{3,5}$ \\ ${ }^{1}$ Division of Pathology, CRO-National Cancer Institute, Aviano, Italy; ${ }^{2}$ Division of Epidemiology and \\ Biostatistics, European Institute of Oncology, Milan, Italy; ${ }^{3}$ Division of Pathology and Laboratory Medicine, \\ European Institute of Oncology, Milan, Italy; ${ }^{4}$ Department of Pathology, University of Verona, Verona, Italy \\ and ${ }^{5}$ University of Milan School of Medicine, Milan, Italy
}

\begin{abstract}
The current TNM classification considers a tumor nodule in the pericolic/perirectal adipose tissue as venous invasion if the nodule has an irregular contour and as regional lymph node metastasis if the nodule has the form and smooth contour of a lymph node. However, detailed studies on the clinico-pathological implications of pericolonic tumor deposits and of extranodal extension are still lacking. We investigated the impact of these metastatic deposits in the pericolic fat in a series of 228 patients with advanced colon cancer. The pericolonic tumor deposits were characterized by their appearance, size, distance from the primary tumor and by their relation with the lymphatic tissue not organized in lymph nodes. These features were then compared with the clinico-pathological characteristics of the tumors and with the patients' survival. All these lesions were associated with reduced disease-free and overall survivals in a univariate analysis, but only pericolonic tumor deposits retained an independent prognostic role in the multivariate analysis. Our findings suggest that pericolonic tumor deposits are a destructive type of venous invasion different from other types of vessel involvement, and that these lesions may rather be included in the $\mathrm{M}$ category for staging purposes.
\end{abstract}

Modern Pathology (2007) 20, 843-855; doi:10.1038/modpathol.3800791; published online 11 May 2007

Keywords: colonic carcinoma; prognosis; pericolonic tumor deposits; vascular invasion; lymphatic invasion; extracapsular tumor spread

Colorectal cancer is one of the most commonly diagnosed human malignancies, ranking fourth in frequency in men and third in women. ${ }^{1}$ Although the prognosis has slightly improved in the last years (survival estimates at 5 years are 65\% in North America and $54 \%$ in Western Europe), ${ }^{1}$ colonic cancer is still a major cause of death worldwide.

The occurrence of lymph node involvement and the number of metastatic lymph nodes are considered to be important prognostic factors in adenocarcinomas of the colon and rectum, allowing the identification of subgroups of patients with different risk for relapse and a shorter survival., ${ }^{2,3}$

In the latest TNM classification, locally advanced adenocarcinoma patients (corresponding to stage III disease) have been divided into three different substages (IIIA-IIIC) according to the depth of tumor

Correspondence: Dr G Pelosi, MD, Divisione di Anatomia Patologica e Medicina di Laboratorio, Istituto Europeo di Oncologia, Via G Ripamonti, 435, I-20141 Milano, Italy.

E-mail: giuseppe.pelosi@ieo.it

Received 17 December 2006; revised 19 March 2007; accepted 27 March 2007; published online 11 May 2007 infiltration through the intestinal wall and the number of metastatic regional lymph nodes. ${ }^{4}$ Whether additional qualitative features, currently not mentioned in the TNM UICC classification, may be relevant to nodal stage has already been addressed, and a few studies have investigated the prognostic role of the volume of metastatic lymph node involvement and of extracapsular tumor spreading. ${ }^{5,6}$ Vascular, lymphatic and perineural invasion have been shown to correlate with a poor prognosis 7,8 but without directly affecting tumor staging. ${ }^{9,10}$

Besides stage III substaging, another change in the latest TNM classification was the reappraisal of pericolonic tumor deposits, which are metastatic adenocarcinoma nodules occurring in the pericolonic and perirectal fat, often in close association with blood vessels. ${ }^{11,12}$ In the previous TNM edition, these nodular lesions of the perivisceral fat were classified exclusively on the basis of their size, independent of their histologic features, and were considered to be discreet tumor disseminations to the perivisceral fat if the diameter was $3 \mathrm{~mm}$ or less in diameter, or lymph node metastases if larger than 
$3 \mathrm{~mm} .{ }^{13}$ In the new TNM classification, it is the shape that defines pericolonic tumor deposits either as frank vascular (venous) invasion if they have an irregular contour, or metastatic lymph nodes if presenting with smooth contour. ${ }^{4}$

Although the prognostic implications of pericolonic tumor deposits in patients with large bowel adenocarcinoma have already been emphasized in previous studies, ${ }^{12,14-19}$ some uncertainties still remain about their actual prevalence, classification and clinical implications. The same holds true for the detection of vascular invasion in colorectal cancer (ranging from 10 to $89.5 \%$ in published studies ${ }^{20}$ ) and its relationship with pericolonic tumor deposits. Two different patterns of vascular invasion have been identified in colorectal cancer: the 'filling' or 'floating' type, ${ }^{20,21}$ if the vascular wall is preserved, ${ }^{22}$ and the 'occlusive' ${ }^{21}$ or 'infiltrating' ${ }^{20}$ type if the vein wall is damaged up to the complete destruction, with a surrounding fibroinflammatory reaction. ${ }^{21,22}$

Among the above-mentioned types of vascular invasion, pericolonic tumor deposits can be included only in the 'infiltrating' type described by Sternberg et al. ${ }^{20}$ In their article, however, these authors did not investigate the clinical implications of these distinct patterns of vascular invasion, since they were aimed at evaluating the detection rate of different types of vascular invasion in stage IV colorectal cancer, comparing their series to the pertinent literature. ${ }^{20}$ Therefore, the present study, dealing with 228 stage III-IV colonic adenocarcinomas, was undertaken to investigate the prognostic implications of pericolonic tumor deposits in comparison with other types of vascular invasion and the clinical significance of extracapsular tumor spreading in lymph node metastases.

\section{Materials and methods}

\section{Patients}

Between 1988 and 1999, 300 consecutive patients with stage III and IV (sixth TNM classification of malignant tumors) advanced colorectal adenocarcinoma, surgically treated at the European Institute of Oncology in Milan and at the University Hospital of Verona, were identified. Of these, 231 were of colonic origin and 69 rectal. Inclusion criteria for entry into the study included the absence of neoadjuvant treatment, age $\leq 85$ years (three patients aged 87, 87 and 92 years were later excluded from the analysis). Because of the different biological, clinico-pathologic and therapeutic implications of rectal carcinoma, in particular, the introduction of the total mesorectal resection in the latter years of the study and the postoperative radiation therapy offered to many such patients, these tumors were eventually excluded from the analysis. All clinical data were extracted from the patients' original charts, the databases of the participating institutions, direct interviews with surviving patients or members of their families or their family physicians, or from death registry offices. The collected data included date of birth, date of surgery, date of last follow-up/death, date of local recurrence, date of first metastatic spreading, site of metastatic spreading (classified as local recurrence, hepatic, abdominal extrahepatic and distant) and the anatomical location of the neoplasm (classified as right, transverse and left colon). Information on overall survival (OS) was available for all patients, with $141(61.8 \%)$ dying of their disease. The mean survival period was 54.2 months (s.d. 43.1; range 0106 months). Recurrent disease information was assessable in 220 (96\%) patients, with a mean time to event of 39 months (s.d. 38.6; range 0-170 months). Information on adjuvant therapy was available for 205 patients (90\%) (115 treated and 89 untreated).

\section{Histopathologic Features}

One single pathologist reviewed all the original H\&E-stained sections from the two participating centers. A subset $(20 \%)$ of cases with pericolonic tumor deposits was then reviewed by a second pathologist. No statistical differences were seen between the recordings of the two observers.

All the original H\&E-stained sections of each case were reviewed, and the following data were collected: confirmation of T3 or T4 status, tumor grade (categorized with a three-tier grading system using the extent of gland formation and microtubular structures as the primary grade criteria $\left.{ }^{23,24}\right)$, occurrence and type of vascular invasion (intramural, extramural or lymphatic), occurrence of perineural invasion, tumor growth margins (expansive or infiltrative), number of metastatic lymph nodes, identification of lymph node extracapsular tumor spreading and number and characteristics of pericolonic tumor deposits. In particular, pericolonic tumor deposits were defined using the current TNM criteria, ${ }^{4}$ so these lesions were distinguished from metastatic lymph nodes, but additional features were recorded, such as lymphocyte infiltration, nodule size (considered as either the maximum or the mean diameter within individual tumors) and distance from the main tumor dichotomized as either $\leq 1 \mathrm{~cm}$ if the pericolonic tumor deposit was included in the same block as the primary tumor (Figure 1a), or $>1 \mathrm{~cm}$ if the pericolonic tumor deposit was found further away in the perivisceral fat.

Pericolonic tumor deposits showed a heterogeneous histologic appearance but shared a common and frequent association with partially destroyed, large muscularized veins (Figure 1b). In general, pericolonic tumor deposits had irregularly lobulated shapes, were encircled by a discontinuous rim of nonparallel collagen fibers of different thickness and were sometimes compartmentalized by fibrous septa (Figure 1d). Typically, there was no necrosis or associated lymphoid tissue (Figure 1a-d). Other 


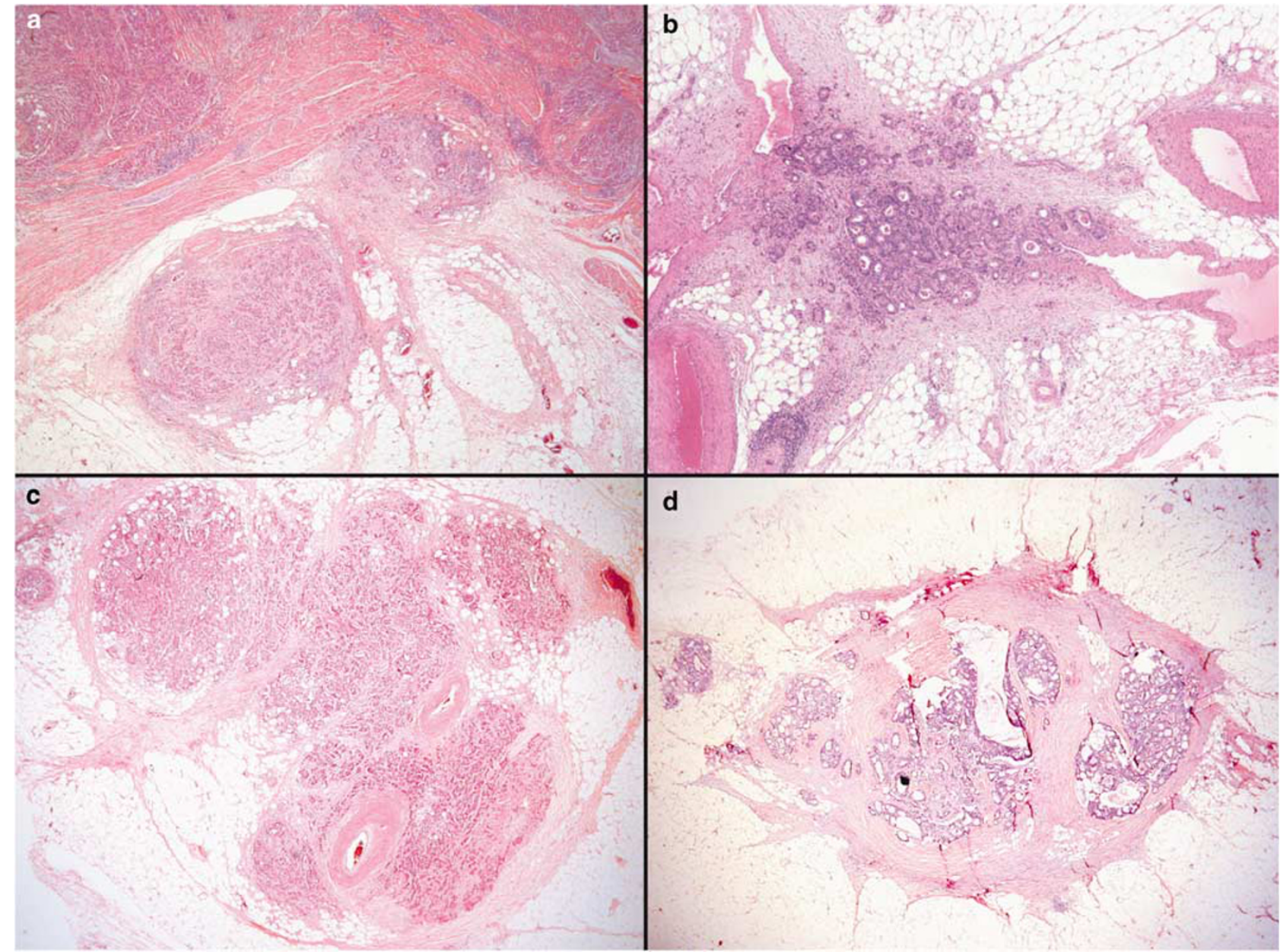

Figure 1 Pericolonic tumor deposits without lymphocytes: there is often a close relation to vascular structures.

pericolonic tumor deposits were still clustered in the perivenous spaces, but showed scattered lymphoid tissue that was not organized in the residual lymph node structures (Figure 2a-d).

Lymph nodes completely replaced by carcinomatous tissue were characterized by roundish contours and a smooth shape with a continuous peripheral capsule of parallel collagen fibers of the same thickness and often showed necrotic areas (Figure 3a-d). In mucinous carcinoma, lymph nodes completely replaced were often filled with mucus (Figure 3c and d). The extracapsular type of lymph node metastasis showed tumor involvement of the perinodal tissue associated with nodal metastases.

\section{Statistical Analysis}

Associations between pericolonic tumor deposits and other clinico-pathological characteristics of the tumor were assessed by the Fisher's exact test for categorical variables and with the Mantel-Haenszel $\chi^{2}$ test for trend for ordinal variables. Disease-free survival (DFS) was calculated from the date of surgery until the date of either loco-regional relapse or the development of distant metastasis. Only mortality from disease was considered for OS analysis. DFS and OS curves were obtained using the Kaplan-Meier method and the comparison between groups was assessed by the log-rank test, considering a $P$-value $<0.05$ as significant. Univariate and multivariate Cox proportional hazard regression were used to assess the prognostic significance of pericolonic tumor deposits and other clinical characteristics on survival. As this study dealt with locally advanced and metastatic tumors that actually have a poor prognosis, patients were censored at 6 years in order to emphasize the prognostic role of pericolonic tumor deposits as a predictive marker for reduced DFS and OS. All tests were two sided. Analyses were performed with the SAS software (Cary, NC).

\section{Results}

\section{Clinico-Pathological Data}

Stage distribution included nine (4\%) tumor patients with stage IIIA, 100 (44\%) with stage IIIB, 


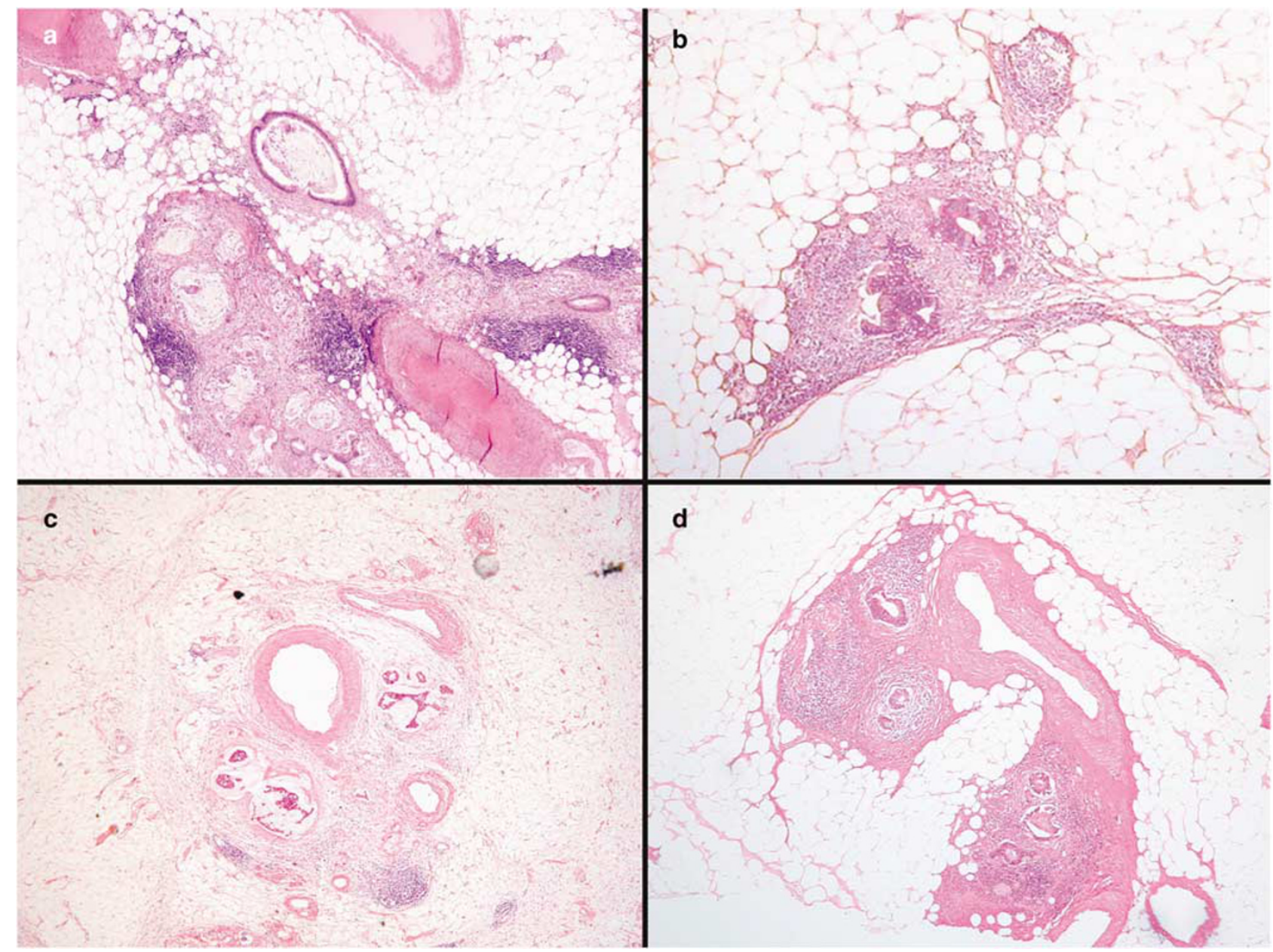

Figure 2 Pericolonic tumor deposits within lymphatic structures without evidence of lymph node, often in the perivascular spaces.

$61(26 \%)$ with stage IIIC and 58 (25\%) with stage IV tumors. Eighty-five patients $(37 \%)$ had right colon carcinoma, $14(6 \%)$ transverse colon carcinoma and 129 (57\%) left colon carcinoma. Twenty patients (9\%) experienced local recurrences and 134 $(59 \%)$ distant metastases, classified as hepatic (80 patients), abdominal extrahepatic (22 patients) and extra-abdominal (32 patients). Sixteen $(7 \%)$ of the adenocarcinomas were grade $1,144(63 \%)$ grade 2 and 68 (30\%) grade 3, with 213 (93\%) of them being typical adenocarcinoma, $13(6 \%)$ mucinous and $2(1 \%)$ signet ring cell adenocarcinoma. Intramural venous vessel invasion was detected in 51 $(22 \%)$ cases, extramural vascular invasion in 69 $(30 \%)$, lymphatic vessel invasion in $21(9 \%)$ and perineural invasion in $29(13 \%)$. There was a prevalence of both G3 tumors and intramural venous invasion in right-sided colon cancer in comparison with that of left-sided ones (for both $P<0.001)$.

The median number of LN examined was 12 (range 1-74), and was similar $(P=0.59)$ in patients with pericolonic tumor deposits (median 12, range 2-38) and those without (median 13, range $1-74)$.

\section{Metastatic Involvement of Perivisceral Fat}

The distribution of venous, lymphatic invasion and pericolonic tumor deposits according to tumor stage is reported in Figure 4. In particular, intramural venous invasion was present in 38 cases $(22 \%)$ of stage III and 13 cases (22\%) of stage IV disease, extramural venous invasion in 44 cases $(25 \%)$ of stage III and 26 cases (45\%) of stage IV disease, lymphatic invasion in 16 cases (9\%) of stage III and five cases $(9 \%)$ of stage IV disease, perineural invasion in 19 cases (11\%) of stage III and 10 cases $(17 \%)$ of stage IV disease, pericolonic tumor deposits with lymphocytes were present in 19 cases $(11 \%)$ of stage III and eight cases $(14 \%)$ of stage IV disease, pericolonic tumor deposits without lymphocytes in 49 cases $(28 \%)$ of stage III and 27 cases $(55 \%)$ of stage IV disease and extracapsular tumor spreading in 69 cases $(40 \%)$ of stage III and 32 cases $(55 \%)$ of stage IV disease.

The two types of pericolonic tumor deposits were definitely separated: their presence was reciprocally exclusive. There was no patient with both types of lesions, except one single case with 10 pericolonic tumor deposits without lymphocytes and 

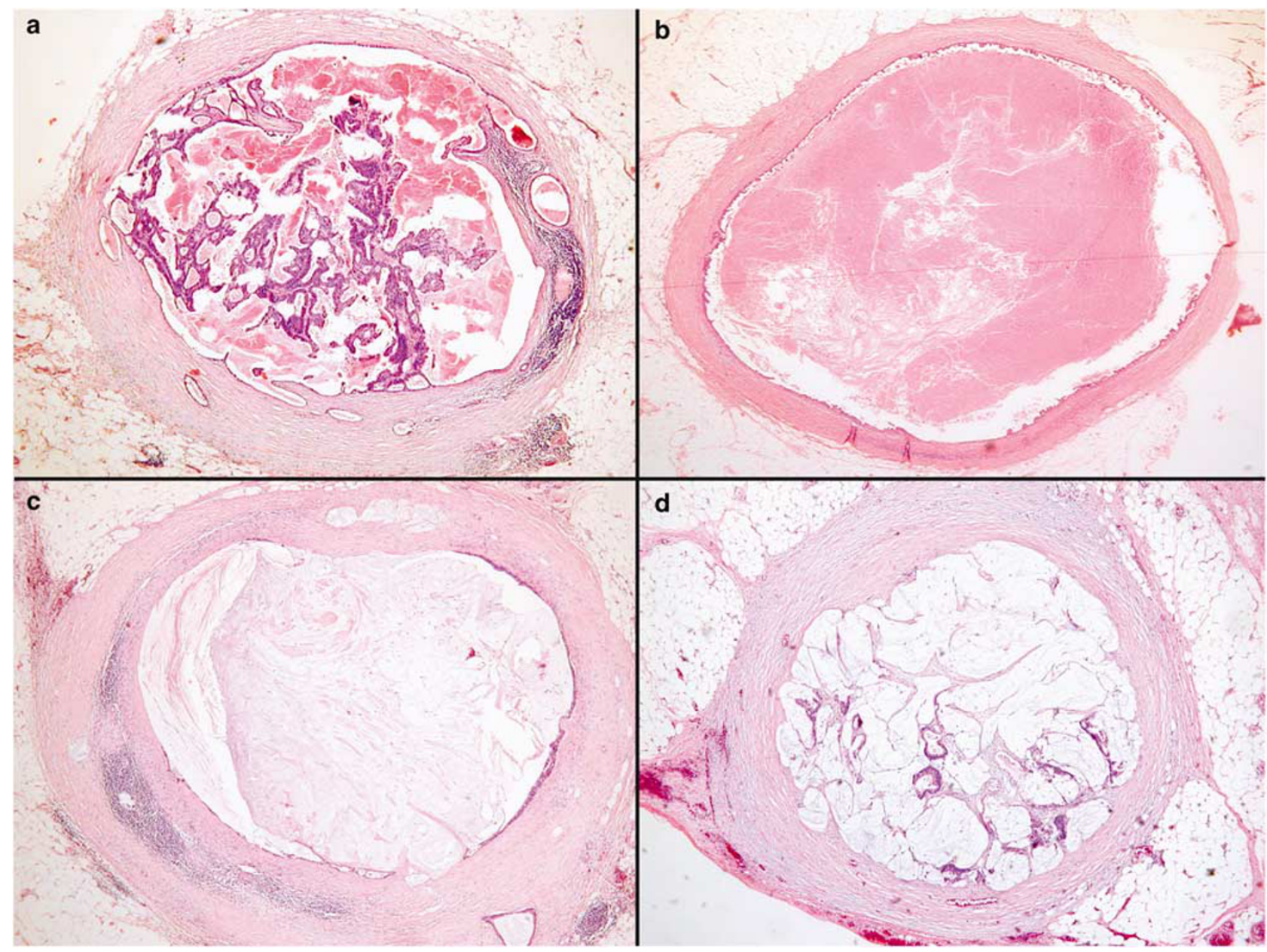

Figure 3 Lymph nodes under complete replacement by metastatic tumor.

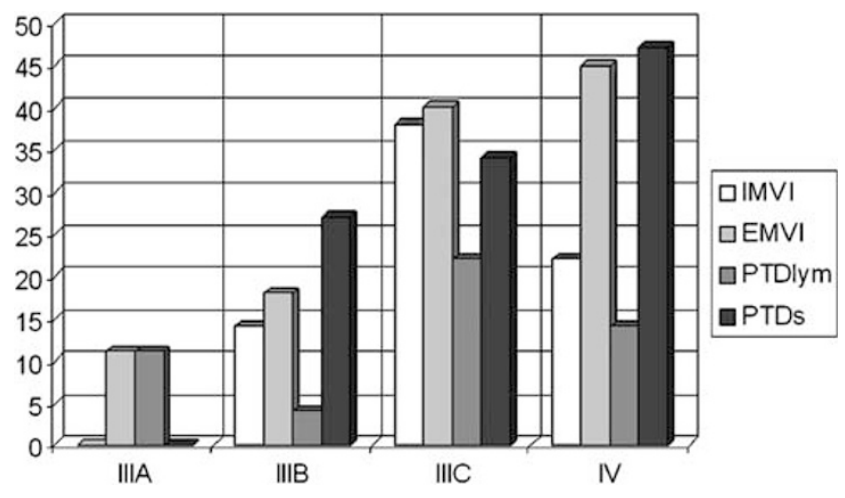

Figure 4 Distribution of intramural venous invasion (IMVI), extramural venous invasion (EMVI), pericolonic tumor deposits with lymphocytes (PTDlym) and pericolonic tumor deposits without lymphocytes (PTDs) throughout stages IIIA-IV.

one single pericolonic tumor deposit with lymphocytes.

The distance of pericolonic tumor deposits from the main tumor was up to $1 \mathrm{~cm}$ in $18(17 \%)$ cases and $>1 \mathrm{~cm}$ in $85(83 \%)$ cases. The size of the largest pericolonic tumor deposit per single case ranged from 0.3 to $22 \mathrm{~mm}$ (mean $=6.0 \mathrm{~mm}$, s.d. $=4.7 \mathrm{~mm}$, median $=4.9 \mathrm{~mm})$, whereas the mean size of all pericolonic tumor deposits was $4.2 \mathrm{~mm}$ (s.d. $=3.1 \mathrm{~mm}$, median $=3.3 \mathrm{~mm}$, range $0.3-15 \mathrm{~mm}$ ).

The occurrence of pericolonic tumor deposits was associated with metastatic disease, without any preferential site distribution. When considering all types of pericolonic tumor deposits, these were correlated with almost all the known adverse prognostic factors, except for mucinous differentiation and lymphatic invasion (Table 1). Conversely, when pericolonic tumor deposits were evaluated according to the presence of lymphocytes, a close association was retained only between the occurrence of pericolonic tumor deposits with lymphocytes and the number of metastatic lymph nodes or the lymphatic invasion. Pericolonic tumor deposits without lymphocytes were equally distributed in tumors with and without vascular invasion (Table 1).

\section{Survival Analysis}

The survival curves of the patients treated in the two participating centres were not statistically different. 
Table 1 Association between lymph node status, extracapsular tumor spreading, presence of pericolonic tumor deposits, and of surrounding lymphocytes and other clinico-pathological characteristics

\begin{tabular}{|c|c|c|c|c|c|c|c|c|c|c|c|c|}
\hline & \multicolumn{3}{|c|}{$p N$} & \multicolumn{3}{|c|}{ ECTS } & \multicolumn{3}{|c|}{ Presence of PTDs (all types) } & \multicolumn{3}{|c|}{ Surrounding lymphocytes } \\
\hline & N1 & N2 & P-value & No & Yes & $\mathrm{P}$-value & No & Yes & P-value & No & Yes & P-value \\
\hline \multicolumn{13}{|l|}{ PTDs } \\
\hline Absent & 92 & 36 & & 86 & 42 & & 128 & - & & - & - & \\
\hline Present & 47 & 53 & 0.0002 & 43 & 57 & 0.0003 & - & 100 & - & 74 & 26 & - \\
\hline \multicolumn{13}{|c|}{ Surrounding lymphocytes } \\
\hline No & 40 & 34 & & 34 & 40 & & - & 74 & & 74 & - & \\
\hline Yes & 7 & 19 & 0.022 & 9 & 17 & 0.36 & - & 26 & - & - & 26 & - \\
\hline \multicolumn{13}{|l|}{ Histology } \\
\hline Non-mucinous & 129 & 84 & & 122 & 91 & & 122 & 91 & & 66 & 25 & \\
\hline Mucinous & 10 & 5 & 0.79 & 7 & 8 & 0.43 & 6 & 9 & 0.28 & 8 & 1 & 0.44 \\
\hline \multicolumn{13}{|l|}{ Grade } \\
\hline G1 & 13 & 3 & & 14 & 2 & & 15 & 1 & & 1 & 0 & \\
\hline G2 & 100 & 44 & & 88 & 56 & & 83 & 61 & & 43 & 18 & \\
\hline G3 & 26 & 42 & $<0.0001$ & 27 & 41 & $<0.0001$ & 30 & 38 & 0.0008 & 30 & 8 & 0.47 \\
\hline \multicolumn{13}{|l|}{$p T$} \\
\hline pT1 & 2 & 0 & & 2 & 0 & & 2 & 0 & & 0 & 0 & \\
\hline pT2 & 11 & 1 & & 7 & 5 & & 10 & 2 & & 0 & 2 & \\
\hline pT3 & 112 & 73 & & 107 & 78 & & 104 & 81 & & 60 & 21 & \\
\hline pT4 & 14 & 15 & 0.0086 & 13 & 16 & 0.12 & 12 & 17 & 0.0071 & 14 & 3 & 0.11 \\
\hline \multicolumn{13}{|l|}{$p N$} \\
\hline N1 & 139 & 0 & & 101 & 38 & & 92 & 47 & & 40 & 7 & \\
\hline N2 & 0 & 89 & - & 28 & 61 & $<0.0001$ & 36 & 53 & 0.0002 & 34 & 19 & 0.022 \\
\hline \multicolumn{13}{|c|}{ Number of positive nodes } \\
\hline 1 & 62 & 0 & & 46 & 16 & & 45 & 17 & & 16 & 1 & \\
\hline 2 & 53 & 0 & & 40 & 13 & & 32 & 21 & & 17 & 4 & \\
\hline $3-5$ & 24 & 44 & & 31 & 37 & & 36 & 32 & & 23 & 9 & \\
\hline$>5$ & 0 & 45 & - & 12 & 33 & $<0.0001$ & 15 & 30 & $<0.0001$ & 18 & 12 & 0.0076 \\
\hline \multicolumn{13}{|l|}{ ECTS } \\
\hline No & 101 & 28 & & 129 & 0 & & 86 & 43 & & 34 & 9 & \\
\hline Yes & 38 & 61 & $<0.0001$ & 0 & 99 & - & 42 & 57 & 0.0003 & 40 & 17 & 0.36 \\
\hline \multicolumn{13}{|l|}{$p M$} \\
\hline Mo & 111 & 59 & & 103 & 67 & & 105 & 65 & & 47 & 18 & \\
\hline M1 & 28 & 30 & 0.029 & 26 & 32 & 0.046 & 23 & 35 & 0.0038 & 27 & 8 & 0.64 \\
\hline Stage & & & & & & & & & & & & \\
\hline IIIA & 9 & 0 & & 6 & 3 & & 8 & 1 & & 0 & 1 & \\
\hline IIIB & 99 & 1 & & 75 & 25 & & 70 & 30 & & 26 & 4 & \\
\hline IIIC & 3 & 58 & & 22 & 39 & & 27 & 34 & & 21 & 13 & \\
\hline IV & 28 & 30 & $<\mathbf{0 . 0 0 0 1}$ & 26 & 32 & $<0.0001$ & 23 & 35 & $<0.0001$ & 27 & 8 & 0.74 \\
\hline Vascular invasion & & & & & & & & & & & & \\
\hline Absent & 95 & 32 & & 85 & 42 & & 88 & 39 & & 28 & 11 & \\
\hline Present & 44 & 57 & $<\mathbf{0 . 0 0 0 1}$ & 44 & 57 & 0.0004 & 40 & 61 & $<\mathbf{0 . 0 0 0 1}$ & 46 & 15 & 0.82 \\
\hline$I M V I$ & & & & & & & & & & & & \\
\hline Absent & 117 & 60 & & 108 & 69 & & 107 & 70 & & 49 & 21 & \\
\hline Present & 22 & 29 & 0.0053 & 21 & 30 & 0.0158 & 21 & 30 & 0.0166 & 25 & 5 & 0.22 \\
\hline EMVI & & & & & & & & & & & & \\
\hline Absent & 109 & 50 & & 99 & 60 & & 103 & 56 & & 42 & 14 & \\
\hline Present & 30 & 39 & 0.0006 & 30 & 39 & 0.0093 & 25 & 44 & $<0.0001$ & 32 & 12 & 0.82 \\
\hline Lymphatic invasic & & & & & & & & & & & & \\
\hline Absent & 135 & 72 & & 121 & 86 & & 120 & 87 & & 69 & 18 & \\
\hline Present & 4 & 17 & $<\mathbf{0 . 0 0 0 1}$ & 8 & 13 & 0.104 & 8 & 13 & 0.1059 & 5 & 8 & 0.0041 \\
\hline Neural invasion & & & & & & & & & & & & \\
\hline Absent & 130 & 69 & & 121 & 78 & & 120 & 79 & & 62 & 17 & \\
\hline Present & 9 & 20 & 0.0008 & 8 & 21 & 0.0011 & 8 & 21 & 0.0012 & 12 & 9 & 0.056 \\
\hline Tumor border & & & & & & & & & & & & \\
\hline Expansive & 7 & 3 & & 7 & 3 & & 8 & 2 & & 2 & 0 & \\
\hline Infiltrative & 132 & 86 & 0.74 & 122 & 96 & 0.52 & 120 & 98 & 0.19 & 72 & 26 & 1.00 \\
\hline Peritumoral inflar & nation & & & & & & & & & & & \\
\hline Absent & 134 & 87 & & 123 & 98 & & 122 & 99 & & 73 & 26 & \\
\hline Present & 5 & 2 & 0.27 & 6 & 1 & 0.097 & 6 & 1 & 0.094 & 1 & 0 & 0.74 \\
\hline
\end{tabular}

PTDs, pericolonic tumor deposits; ECTS, extracapsular tumor spreading; IMVI, intramural venous invasion; EMVI, extramural vascular invasion. Bold $=P<0.05$. 
Table 2 Univariate analysis for disease-free and overall survival

\begin{tabular}{|c|c|c|c|c|}
\hline \multirow[t]{2}{*}{ Univariate analysis } & \multicolumn{2}{|c|}{ Disease-free survival } & \multicolumn{2}{|c|}{ Overall survival (dead from cancer) } \\
\hline & HR $(95 \% C I)$ & P-value & HR $(95 \% C I)$ & P-value \\
\hline \multicolumn{5}{|l|}{ ECTS } \\
\hline No & 1.00 & & 1.00 & \\
\hline Yes & $1.79(1.31-2.45)$ & 0.0003 & $1.79(1.28-2.50)$ & 0.0007 \\
\hline \multicolumn{5}{|l|}{ PTDs } \\
\hline Absent & 1.00 & & 1.00 & \\
\hline Present & $3.22(2.33-4.47)$ & $<0.0001$ & $3.69(2.60-5.24)$ & $<\mathbf{0 . 0 0 0 1}$ \\
\hline \multicolumn{5}{|l|}{ PTDs number } \\
\hline None & 1.00 & & 1.00 & \\
\hline 1 & $2.40(1.59-3.61)$ & $<0.0001$ & $3.10(2.00-4.79)$ & $<0.0001$ \\
\hline 2 & $3.64(2.12-6.24)$ & $<0.0001$ & $3.18(1.81-5.58)$ & $<0.0001$ \\
\hline $3+$ & $4.81(3.15-7.34)$ & $<0.0001$ & $5.07(3.27-7.87)$ & $<0.0001$ \\
\hline \multicolumn{5}{|l|}{ Presence of lymphocytes } \\
\hline No PTDs & 1.00 & & 1.00 & \\
\hline PTDs with lymphocytes & $2.85(1.74-4.67)$ & $<0.0001$ & $2.57(1.53-4.32)$ & 0.0003 \\
\hline PTDs without lymphocytes & $3.36(2.38-4.76)$ & $<0.0001$ & $4.30(2.96-6.25)$ & $<\mathbf{0 . 0 0 0 1}$ \\
\hline \multicolumn{5}{|l|}{ PTDs maximum size } \\
\hline No PTDs & 1.00 & & 1.00 & \\
\hline$<2 \mathrm{~mm}$ & $2.03(1.17-3.52)$ & 0.012 & $2.07(1.17-3.69)$ & 0.013 \\
\hline $2-5 \mathrm{~mm}$ & $3.17(2.09-4.82)$ & $<0.0001$ & $3.93(2.52-6.15)$ & $<.0001$ \\
\hline$>5 \mathrm{~mm}$ & $4.48(2.97-6.75)$ & $<0.0001$ & $5.15(3.35-7.93)$ & $<\mathbf{0 . 0 0 0 1}$ \\
\hline \multicolumn{5}{|l|}{ PTDs median size } \\
\hline No PTDs & 1.00 & & 1.00 & \\
\hline$<2 \mathrm{~mm}$ & $2.49(1.54-4.02)$ & 0.0002 & $2.74(1.66-4.52)$ & $<0.0001$ \\
\hline $2-5 \mathrm{~mm}$ & $3.35(2.27-4.94)$ & $<0.0001$ & $3.87(2.56-5.86)$ & $<0.0001$ \\
\hline$>5 \mathrm{~mm}$ & $4.26(2.62-6.95)$ & $<0.0001$ & $5.17(3.10-8.62)$ & $<\mathbf{0 . 0 0 0 1}$ \\
\hline \multicolumn{5}{|l|}{ Localization } \\
\hline Left & 1.00 & & 1.00 & \\
\hline Right-transverse & $1.42(1.04-1.95)$ & 0.029 & $1.44(1.03-2.01)$ & 0.033 \\
\hline \multicolumn{5}{|l|}{ Histology } \\
\hline Non-mucinous & 1.00 & & 1.00 & \\
\hline Mucinous & $1.66(0.94-2.94)$ & 0.083 & $2.11(1.19-3.75)$ & 0.011 \\
\hline \multicolumn{5}{|l|}{ Grade } \\
\hline G1 & 1.00 & & 1.00 & \\
\hline G2 & $2.05(0.96-4.42)$ & 0.083 & $2.44(0.97-6.01)$ & 0.052 \\
\hline G3 & $2.43(1.10-5.37)$ & 0.029 & $2.96(1.17-7.49)$ & 0.022 \\
\hline \multicolumn{5}{|l|}{$p T$} \\
\hline pT1-2 & 1.00 & & 1.00 & \\
\hline pT3-4 & $4.01(1.48-10.8)$ & 0.0062 & $2.93(1.08-7.92)$ & 0.034 \\
\hline \multicolumn{5}{|l|}{$p N$} \\
\hline N1 & 1.00 & & 1.00 & \\
\hline N2 & $1.74(1.27-2.39)$ & 0.0006 & $1.56(1.11-2.19)$ & 0.0096 \\
\hline \multicolumn{5}{|l|}{ Number of positive nodes } \\
\hline 1 & 1.00 & & 1.00 & \\
\hline $2-5$ & $1.65(1.10-2.45)$ & 0.015 & $1.59(1.03-2.46)$ & 0.035 \\
\hline$>5$ & $2.91(1.82-4.63)$ & $<\mathbf{0 . 0 0 0 0 1}$ & $3.30(2.03-5.37)$ & $<\mathbf{0 . 0 0 0 1}$ \\
\hline \multicolumn{5}{|l|}{$p M$} \\
\hline Mo & 1.00 & & 1.00 & \\
\hline M1 & $3.67(2.62-5.15)$ & $<0.0001$ & $5.93(4.13-8.52)$ & $<0.0001$ \\
\hline Stage & & & & \\
\hline IIIA & $0.30(0.07-1.12$ & 0.091 & $0.43(0.11-1.77)$ & 0.24 \\
\hline IIIB & 1.00 & & 1.00 & \\
\hline IIIC & $1.75(1.17-2.63)$ & 0.0065 & $1.62(1.04-2.52)$ & 0.033 \\
\hline IV & $4.31(2.94-6.32)$ & $<0.0001$ & $6.92(4.56-10.5)$ & $<0.0001$ \\
\hline$I M V I$ & & & & \\
\hline Absent & 1.00 & & 1.00 & \\
\hline Present & $1.34(0.93-1.93)$ & 0.12 & $1.56(1.07-2.29)$ & 0.022 \\
\hline$E M V I$ & & & & \\
\hline Absent & 1.00 & & 1.00 & \\
\hline Present & $1.74(1.25-2.42)$ & 0.0010 & $1.63(1.14-2.32)$ & 0.0069 \\
\hline
\end{tabular}

PTDs, pericolonic tumor deposits; ECTS, extracapsular tumor spreading; IMVI, intramural venous invasion; EMVI, extramural vascular invasion. No association was found between survival and center, sex, age, lymphatic and neural invasion, tumor border, peritumoral lymphocytic infiltration and adjuvant treatment.

Bold $=P<0.05$. 
Traditional prognostic variables such as $\mathrm{pT}, \mathrm{pN}$, tumor grade and vascular invasion strongly correlated with patients' life expectancy (Table 2 and Figure 6), with TNM tumor staging being the best predictor of survival (Figure 5). A correlation with poorer clinical outcome was also noted for mucinous differentiation, right tumor location and extracapsular tumor spreading (Table 2 and Figure 6). A significant relationship was found between a shorter survival rate and any type of pericolonic tumor deposits, as well as with their increasing number and size (Table 2; Figures 7 and 8). Stratifying the prognostic impact of pericolonic tumor deposits according to tumor stage, it emerged that the occurrence of pericolonic tumor deposits without lymphocytes was detrimental for survival in all tumor substages (IIIA-IIIC), whereas pericolonic tumor deposits with lymphocytes affected prognosis only for patients with stage IIIA and IIIB diseases (Figure 7).

In the multivariate analysis, pericolonic tumor deposits with and without lymphocytes emerged as an independent prognostic factor for both OS and DFS (Table 3, model 1), together with tumor stage and tumor localization. Intramural venous invasion,

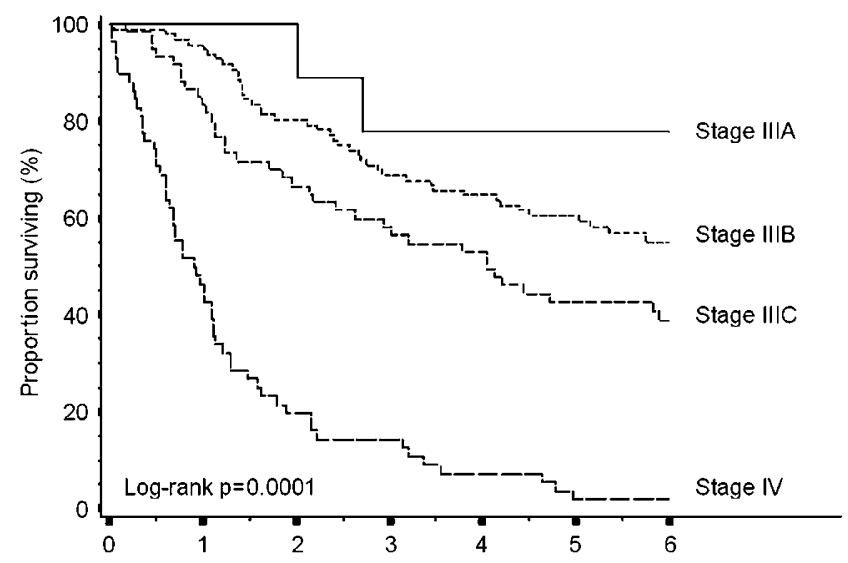

Figure 5 Overall survival according to tumor stage.

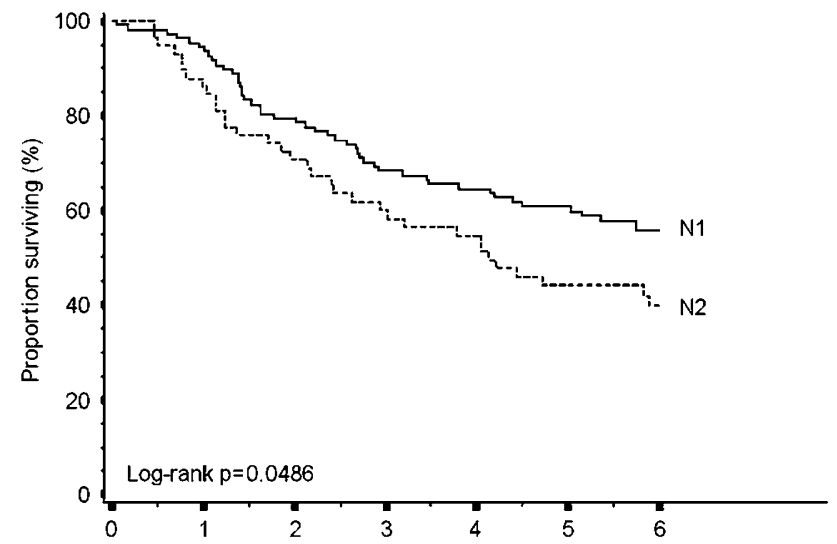

extramural venous invasion, lymphatic invasion and tumor grade were not independent prognostic factors when adjusted for the presence of any type of pericolonic tumor deposits.

Adjusting then the type of pericolonic tumor deposits (with and without lymphocytes) for size and number, the relevance to prognosis of the latter emerged. In particular, when considering the combined features of pericolonic tumor deposits, both the number (two or more) and the absence of lymphocytes, but not the size, were associated with a worse prognosis (Table 3, model 2).

In a multivariate analysis adjusted for other clinical and pathological characteristics including the presence of pericolonic tumor deposits, extracapsular tumor spreading in lymph node metastases did not emerged as an independent prognostic factor (Table 3). However, in a subset analysis limited to patients with stage III colon cancer, the independent prognostic value of extranodal extension for DFS was of borderline significance $(\mathrm{HR}=1.58,95 \%$ $\mathrm{CI}=0.95-2.61, P=0.076)$.

\section{Discussion}

We performed this study to examine the clinicopathological implications of different metastatic occurrences that are commonly encountered in routine histopathological examination of pericolonic fat in advanced-stage large bowel adenocarcinoma specimens.

With regard to the TNM staging system, we paid attention to features that have been considered differently in the subsequent editions (fifth and sixth), such as pericolonic tumor deposits, or not taken into consideration, such as the extracapsular tumor spreading.

The currently adopted TNM staging system for colorectal adenocarcinoma is based mainly upon the assessment of the anatomic extent of the disease at the time of diagnosis, although one of its main objectives is to provide prognostic indications for

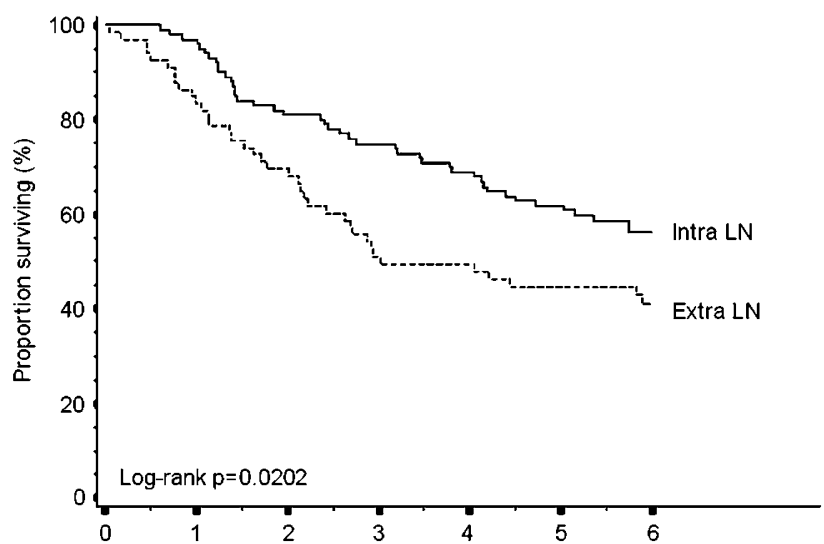

Figure 6 Overall survival according to nodal status (left) and extracapsular tumor spreading (right) in patients with stage III colon cancer. 

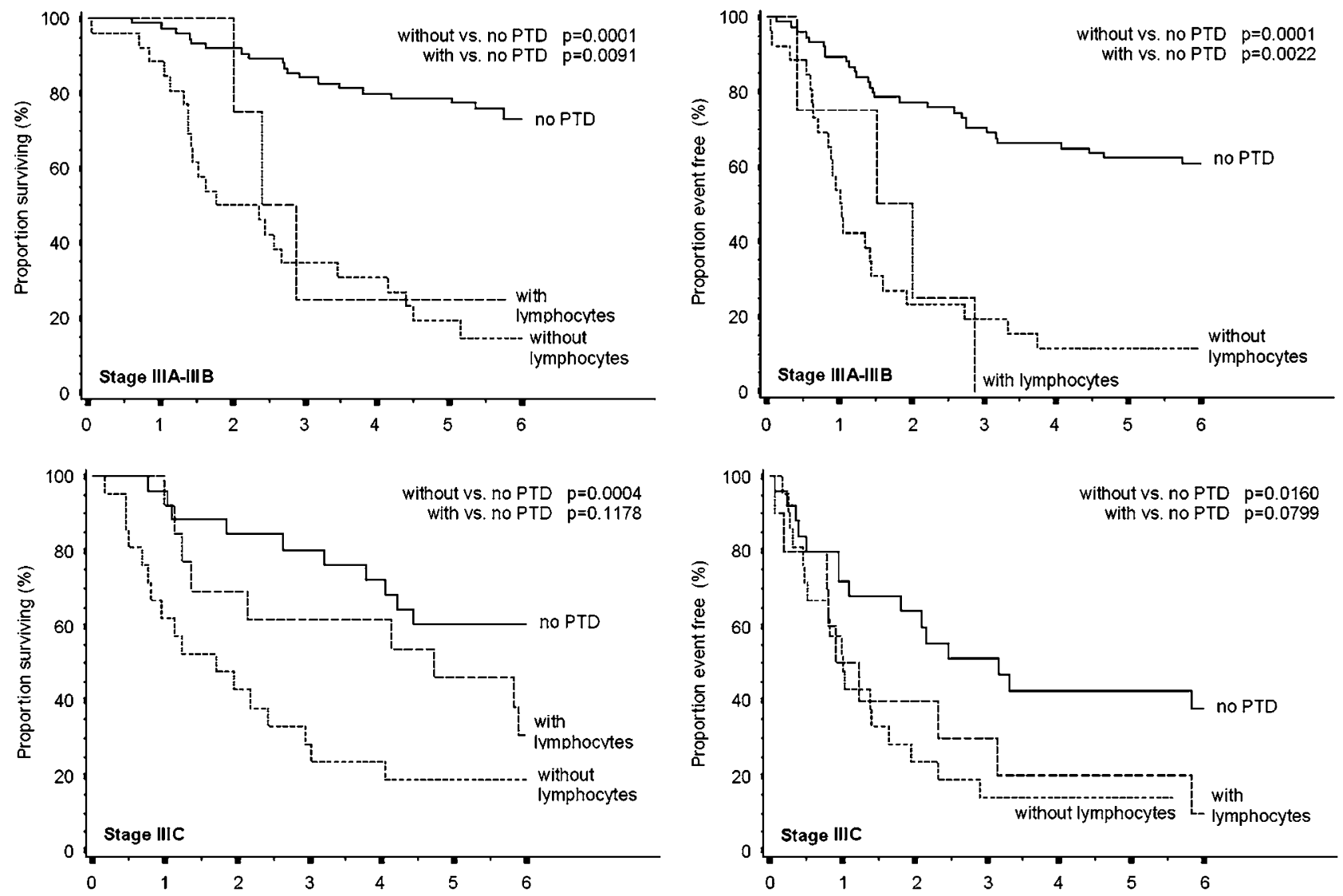

Figure 7 OS (left) and DFS (right) according to the presence and type of pericolonic tumor deposits (with or without lymphocytes) in patients in stage IIIA-IIIB (top) and stage IIIC (bottom) colon cancer.

the patients' best treatment. ${ }^{4} \mathrm{Up}$ to one half of patients with locally advanced colorectal cancer relapse, ${ }^{25,26}$ mostly within the first 3 years of followup. $^{27}$ Therefore, the prognostic heterogeneity of stage III colorectal cancer $^{28}$ has been addressed by stratifying the patients into three substages (IIIA-IIIC), adopting criteria based on the depth of the intestinal wall involvement and the number of metastatic lymph nodes. ${ }^{2}$ Missing additional features, however, may account for remarkable prognostic differences between patients in the same risk category. In this regard, the volume of metastatic lymph node involvement is not an independent prognostic indicator in colorectal cancer, ${ }^{5}$ and the role of extracapsular tumor spreading still awaits further confirmation. ${ }^{6}$ As far as vascular invasion is concerned, it is still unclear whether different types of vascular invasion (lymphatic or venous) are prognostically relevant as independent factors ${ }^{10}$ or whether vascular invasion per se may have clinical implications on tumor staging. Vascular invasion, however, plays a pivotal role in the pathology report of colorectal cancer, and this study was specifically aimed at addressing the different implications of diverse types of vascular invasion and at reappraising the role of extracapsular tumor spreading.

One of the most difficult tasks in assessing the clinical implications of pericolonic tumor deposits in colorectal cancer, whose prevalence ranges from 6 to $64 \%$ in the mesorectum, ${ }^{19}$ derives from the observation that these nodular lesions have often been mixed with other types of venous ${ }^{14,16,18,19}$ or lymphatic invasion, ${ }^{18,19}$ lymph node metastasis, ${ }^{19}$ nerve infiltration ${ }^{11,12,14,16,18,19}$ or otherwise non-specified infiltration of the perivisceral fat, ${ }^{14,16,18}$ thus making it difficult to compare data from different studies. As indicated in the last TNM classification, it is the shape of pericolonic tumor deposits, which has become a unique diagnostic clue, but this is not sufficient to consistently distinguish different types of tumor involvement of the perivisceral fat, and so we relied on additional and more stringent histologic criteria, including the thickness and the disposition of the collagen fibers at the periphery of the nodules, the presence of non-organized lymphoid tissue, and the occurrence of necrosis or of fibrous septa inside the nodules.

Among different types of pericolonic tumor deposits, we were especially puzzled by tumor nodules surrounded by lymphocytes that we found in $11 \%$ of stage III and $14 \%$ of stage IV tumors, as opposed to pericolonic tumor deposits without lymphocytes that we observed in $28 \%$ of stage III and $55 \%$ of stage IV tumors. As lymphatic invasion has been referred to as either the invasion of thin-walled vessels within the colonic wall ${ }^{8}$ or the 

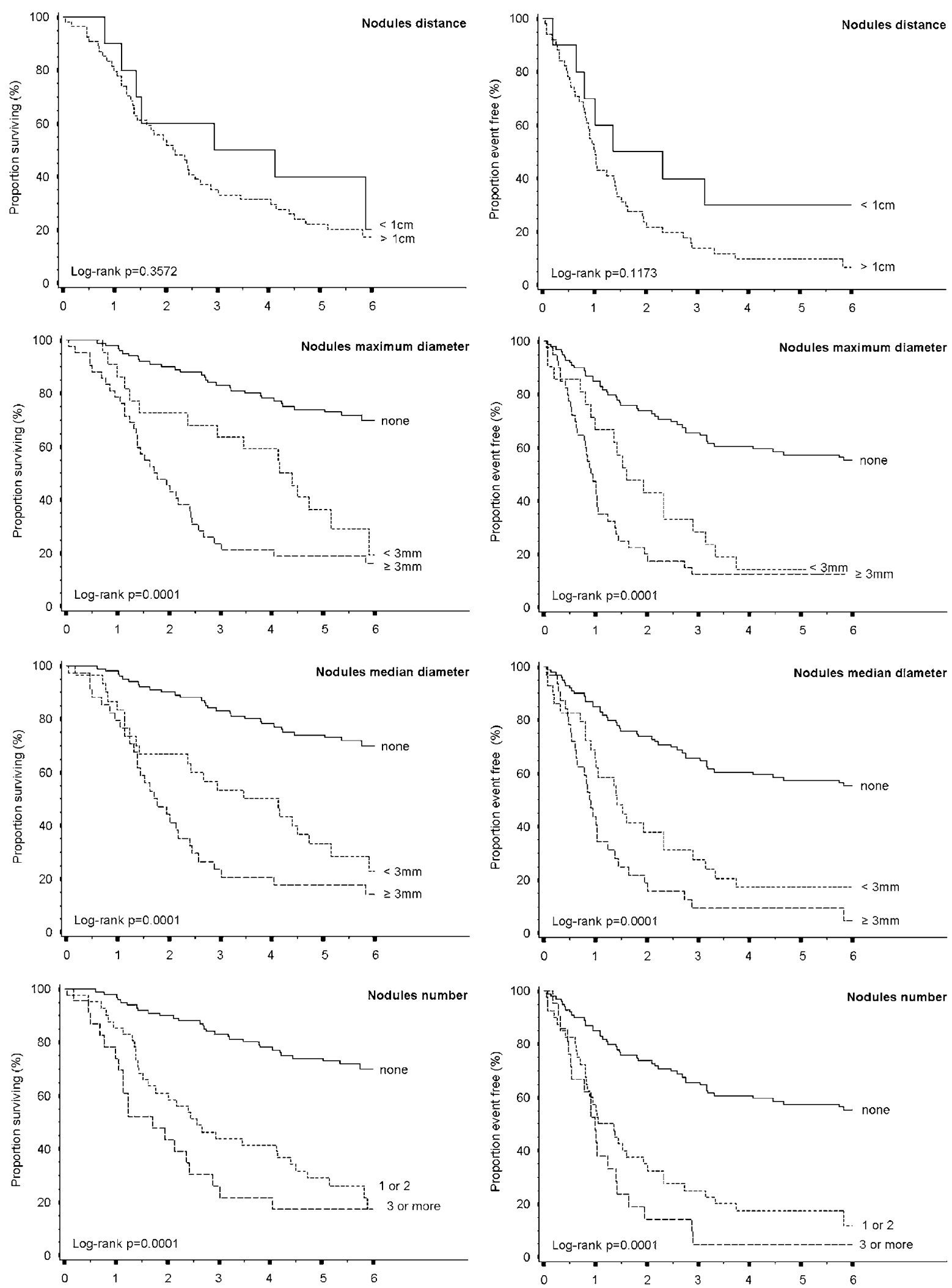

Figure 8 OS (left) and DFS (right) according to nodule distance, median and maximum diameter and nodules number in patients with stage III colon cancer. 
Table 3 Multivariate analysis for disease-free and overall survival

\begin{tabular}{|c|c|c|c|c|c|}
\hline & & \multicolumn{2}{|c|}{ Disease-free survival } & \multicolumn{2}{|c|}{ Overall survival (dead from cancer) } \\
\hline & & $H R(95 \% C I)^{*}$ & $\mathrm{P}$-value* & $H R(95 \% C I)^{*}$ & $\mathrm{P}$-value* \\
\hline \multicolumn{6}{|l|}{ Model 1} \\
\hline ECTS & Yes vs no & $1.40(0.98-1.99)$ & 0.067 & $1.15(0.77-1.72)$ & 0.49 \\
\hline \multirow[t]{2}{*}{ PTDs } & PTDs with lymphocytes vs no PTD & $1.96(1.11-3.47)$ & 0.021 & $2.10(1.14-3.85)$ & 0.017 \\
\hline & PTDs without lymphocytes vs no PTD & $2.87(1.95-4.24)$ & $<0.0001$ & $4.00(2.61-6.13)$ & $<\mathbf{0 . 0 0 0 1}$ \\
\hline Localization & Right/transverse & $1.63(1.14-2.32)$ & $\mathbf{0 . 0 0 7}$ & $1.75(1.19-2.57)$ & 0.005 \\
\hline Histology & Mucinous vs non-mucinous & $1.26(0.68-2.33)$ & 0.47 & $1.70(0.92-3.13)$ & 0.09 \\
\hline \multirow[t]{2}{*}{ Grade } & G2 vs G1 & $0.85(0.35-2.07)$ & 0.77 & $0.94(0.32-2.78)$ & 0.91 \\
\hline & G3 vs G1 & $0.61(0.23-1.62)$ & 0.32 & $0.65(0.20-2.05)$ & 0.46 \\
\hline \multirow[t]{3}{*}{ Stage } & IIIA vs IIIB & $0.45(0.10-1.90)$ & 0.27 & $0.86(0.19-3.78)$ & 0.84 \\
\hline & IIIC vs IIIB & $1.49(0.93-2.39)$ & 0.09 & $1.51(0.89-2.58)$ & 0.13 \\
\hline & IV vs IIIB & $3.71(2.38-5.78)$ & $<0.0001$ & $6.43(3.99-10.3)$ & $<\mathbf{0 . 0 0 0 1}$ \\
\hline \multicolumn{6}{|l|}{ Model 2} \\
\hline ECTS & Yes vs no & $1.27(0.87-1.85)$ & 0.21 & $0.95(0.62-1.46)$ & 0.82 \\
\hline \multirow[t]{4}{*}{ PTDs } & Two or more PTD's & $2.54(1.50-4.27)$ & 0.0005 & $2.03(1.20-3.44)$ & 0.0083 \\
\hline & Max PTD size $>5 \mathrm{~mm}$ & $1.26(0.77-2.08)$ & 0.36 & $1.63(0.97-2.73)$ & 0.06 \\
\hline & PTDs with lymphocytes vs no PTD & $1.29(0.70-2.39)$ & 0.41 & $1.38(0.72-2.64)$ & 0.33 \\
\hline & PTDs without lymphocytes vs no PTD & $1.99(1.25-3.17)$ & 0.0036 & $2.92(1.76-4.84)$ & $<\mathbf{0 . 0 0 0 1}$ \\
\hline Localization & Right/transverse & $1.64(1.15-2.34)$ & 0.0066 & $1.90(1.28-2.83)$ & 0.0015 \\
\hline Histology & Mucinous vs non-mucinous & $1.08(0.58-2.01)$ & 0.80 & $1.48(0.80-2.74)$ & 0.21 \\
\hline \multirow[t]{2}{*}{ Grade } & $\mathrm{G} 2$ vs $\mathrm{G} 1$ & $0.84(0.34-2.03)$ & 0.69 & $0.88(0.30-2.58)$ & 0.81 \\
\hline & G3 vs G1 & $0.59(0.22-1.57)$ & 0.29 & $0.68(0.21-2.16)$ & 0.51 \\
\hline \multirow[t]{3}{*}{ Stage } & IIIA vs IIIB & $0.42(0.10-1.79)$ & 0.24 & $0.79(0.18-3.47)$ & 0.75 \\
\hline & IIIC vs IIIB & $1.48(0.92-2.39)$ & 0.10 & $1.67(0.95-2.93)$ & 0.07 \\
\hline & IV vs IIIB & $4.30(2.74-6.74)$ & $<0.0001$ & $8.02(4.90-13.2)$ & $<\mathbf{0 . 0 0 0 1}$ \\
\hline
\end{tabular}

PTDs, pericolonic tumor deposits; ECTS, extracapsular tumor spreading.

*Also adjusted for age, sex, year of surgery and other tumor characteristics.

Bold $=P<0.05$.

involvement of afferent lymphatic vessels in regional lymph nodes, ${ }^{10}$ we distinctly separated pericolonic tumor deposits with lymphocytes (but not exhibiting residual features of true lymph nodes, such as a fibrous capsule ${ }^{12}$ ) from the usual lymphatic invasion (Figure 2). We hypothesized that those pericolonic tumor deposits with lymphocytes may represent either an immune reaction to adenocarcinoma cells (as observed at the deepest invasive edges of primary tumors ${ }^{29}$ ), 'vascular invasionassociated changes' (as described in other adenocarcinoma types ${ }^{30}$ ), or de novo formed tertiary lymphoid tissue that is able to facilitate tumor metastatic spread. ${ }^{31}$

To our knowledge, we are the first to document that pericolonic tumor deposits with lymphocytes are associated with a shorter survival time both in the univariate and the multivariate analyses (Tables 2 and 3 , model 1), and that they most likely represent a peculiar pattern of lymphatic invasion, due to their significant correlation with the occurrence and the extent of lymph node metastases and with the occurrence of lymphatic invasion (Table 1). Conversely, pericolonic tumor deposits without lymphocytes were not related to lymph node involvement and could be indicative of a relationship with other types of metastatization, most likely occurring through venous vessels. From this viewpoint, we were not surprised that there were almost no patients with both lesions, and that the occur- rence of pericolonic tumor deposits without lymphocytes increased constantly with the increase in tumor stage (IIIA-IV), similar to that found in extramural venous invasion (Figure 4).

An unexpected finding was the similar distribution of pericolonic tumor deposits without lymphocytes in tumors irrespective of the occurrence of vascular invasion (Table 1), suggesting that these lesions are not a consequence of widespread vascular embolization but could be a de novo, distinct pattern of destructive, metastatic invasion involving vascular and perivascular structures. ${ }^{11,12}$ With regard to the location of pericolonic tumor deposits, our results show that they are mostly encountered between metastatic lymph nodes, but the clinical implications of pericolonic tumor deposits lying close to primary tumors are similar to those of distant nodules (Figure 8).

With regard to nodule size and number, both features influenced OS and DFS in the univariate analysis (Table 2 and Figure 8). Only the number of pericolonic tumor deposits (two or more) and the absence of lymphocytes, however, emerged as independent factors in the multivariate analysis (Table 3, model 2).

The current TNM classification states that vascular (venous) invasion has to be considered among those pT-related qualitative features that are able to predict a poor prognosis but unable to affect the stage.$^{9,10}$ More recently, a proposal has been put 
forward to include venous invasion among stageinfluencing factors in colorectal cancer, ${ }^{32-34}$ similar to renal, hepatic and testicular tumors. ${ }^{4}$

In the literature, there are conflicting data on the general prognostic role of venous invasion, ${ }^{7,10}$ whose prevalence ranges from $10 \%$ up to $90 \%$ of colorectal cancer specimens. ${ }^{20}$ This is most likely due to the different criteria used for its identification or to a different patient selection. In some studies for instance, no distinction was made between venous and lymphatic vessels, ${ }^{7,10}$ between intramural venous invasion and extramural venous invasion or colonic and rectal localization of the primaries. ${ }^{35} \mathrm{In}$ this regard, in the series studied for vascular invasion, identifying the type of vascular invasion (intramural vs extramural), it was found that the extramural type was most predictive of survival. ${ }^{22,36}$ Moreover, among the three patterns of vascular invasion ('filling', 'floating' and 'occlusive'), ${ }^{21}$ the latter type with the fibro-inflammatory reaction may even be protective against subsequent recurrent disease, reducing the likelihood of distant metastases $^{21}$ and has been shown to be associated with a better OS. ${ }^{22}$

As we did not observe any independent relationship between different types of vascular invasion and survival when occurring in association with pericolonic tumor deposits (Table 3), we conclude that pericolonic tumor deposits may actually represent a distinct and more structured type of extravascular vein invasion (much more efficient than the simple tumor cell embolization ${ }^{37}$ ), and that their occurrence should be kept separated from other types of vascular invasion in the pathology report. Pericolonic tumor deposits without lymphocytes might represent a form of in transit metastases of colonic carcinomas, worth being classified as pM1 by virtue of their remarkable impact on patients' prognosis, rather than simple vascular (venous) invasion among pT-related 'optional descriptors', ${ }^{4}$ as previously suggested by Goldstein. ${ }^{12}$

In conclusion, our study highlights the different patho-biological significance and different prognostic weight of pericolonic tumor deposits with regard to other types of vascular invasion in advanced colon cancer. In the analysis limited to stage III colon cancer, the prognostic value of extralymphonodal capsular spreading was of borderline significance as an independent prognostic factor; therefore, further studies enrolling more patients with locally advanced disease are needed to investigate this issue.

\section{Acknowledgements}

This research was supported by the AIRC (Associazione Italiana per la Ricerca sul Cancro). The authors gratefully thank Mrs Anna Maria Colussi for her assistance with editing and $\mathrm{Mr}$ Roberto Biancat for his help in collecting survival data.

\section{References}

1 Parkin DM, Bray F, Ferlay J, et al. Global cancer statistics, 2002. CA Cancer J Clin 2005;55:74-108.

2 Greene FL, Stewart AK, Norton HJ. A new TNM staging strategy for node-positive (stage III) colon cancer: an analysis of 50,042 patients. Ann Surg 2002;236: 416-421.

3 Compton C, Fenoglio-Preiser CM, Pettigrew N, et al. American Joint Committee on Cancer Prognostic Factors Consensus Conference: Colorectal Working Group. Cancer 2000;88:1739-1757.

4 Sobin LH, Wittekind Ch (eds). TNM Classification of Malignant Tumors, 6th edn. Wiley-Liss: New York, 2002.

5 Wong JH, Steinemann S, Tom P, et al. Volume of lymphatic metastases does not independently influence prognosis in colorectal cancer. J Clin Oncol 2002;20:1506-1511.

6 Komuta K, Okudaira S, Haraguchi M, et al. Identification of extracapsular invasion of the metastatic lymph nodes as a useful prognostic sign in patients with resectable colorectal cancer. Dis Colon Rectum 2001;44:1838-1844

7 Compton CC. Colorectal carcinoma: diagnostic, prognostic, and molecular features. Mod Pathol 2003;16: 376-388.

8 Compton CC. Colon and Rectum-Protocol of Examination-CAP Approved, Based on AJCC/UICC TNM, 6th edn. www.cap.org/apps/docs/cancer_protocols/ 2005/colonrectum05_ckw.pdf. 2005. 10-10-2006.

9 Sobin LH. TNM, sixth edition: new developments in general concepts and rules. Sem Surg Oncol 2003; 21:19-22.

10 Compton CC, Greene FL. The staging of colorectal cancer: 2004 and beyond. CA Cancer J Clin 2004; 54:295-308.

11 Stiles TE, Dean P, Harrison J. Nature and significance of nodular metastases of colonic carcinoma in pericolonic fat [abstract]. Mod Pathol 1995;8:69A.

12 Goldstein NS, Turner JR. Pericolonic tumor deposits in patients with $\mathrm{T} 3 \mathrm{~N}+\mathrm{MO}$ colon adenocarcinomas: markers of reduced disease free survival and intraabdominal metastases and their implications for TNM classification. Cancer 2000;88:2228-2238.

13 Sobin LH, Wittekind CH (eds). TNM Classification of Malignant Tumors, 5th edn. Wiley-Liss: New York, 1997.

14 Ueno H, Mochizuki H. Clinical significance of extrabowel skipped cancer infiltration in rectal cancer. Surg Today 1997;27:617-622.

15 Ueno H, Mochizuki H, Tamakuma S. Prognostic significance of extranodal microscopic foci discontinuous with primary lesion in rectal cancer. Dis Colon Rectum 1998;41:55-61.

16 Tateishi S, Arima S, Futami K, et al. A clinicopathological investigation of 'tumor nodules' in colorectal cancer. Surg Today 2005;35:377-384.

17 Singh AK, Myerson RJ, Birnbaum EH, et al. Outcome of patients with rectal adenocarcinoma and localized pelvic non-nodal metastatic foci. Dis Colon Rectum 2000;43:1217-1221.

18 Ratto C, Ricci R, Rossi C, et al. Mesorectal microfoci adversely affect the prognosis of patients with rectal cancer. Dis Colon Rectum 2002;45:733-742.

19 Ono C, Yoshinaga K, Enomoto M, et al. Discontinuous rectal cancer spread in the mesorectum and the 
optimal distal clearance margin in situ. Dis Colon Rectum 2002;45:744-749.

20 Sternberg A, Amar M, Alfici R, et al. Conclusions from a study of venous invasion in stage IV colorectal adenocarcinoma. J Clin Pathol 2002;55:17-21.

21 Ouchi K, Sugawara T, Ono H, et al. Histologic features and clinical significance of venous invasion in colorectal carcinoma with hepatic metastasis. Cancer 1996;78:2313-2317.

22 Talbot IC, Ritchie S, Leighton $\mathrm{M}$, et al. Invasion of veins by carcinoma of rectum: method of detection, histological features and significance. Histopathology 1981;5:141-163.

23 Jass JR, Atkin WS, Cuzick J, et al. The grading of rectal cancer: historical perspectives and a multivariate analysis of 447 cases. Histopathology 1986;10:437-459.

24 Grinnell RS. The grading and prognosis of carcinoma of the colon and rectum. Ann Surg 1939;109:500-533.

25 Obrand DI, Gordon PH. Incidence and patterns of recurrence following curative resection for colorectal carcinoma. Dis Colon Rectum 1997;40:15-24.

26 Gramont A. Adjuvant therapy of stage II and III colon cancer. Semin Oncol 2005;32:11-14.

27 Sadahiro S, Suzuki T, Ishikawa K, et al. Recurrence patterns after curative resection of colorectal cancer in patients followed for a minimum of 10 years. Hepatogastroenterology 2003;50:1362-1366.

28 Merkel S, Mansmann U, Papadopoulos T, et al. The prognostic inhomogeneity of colorectal carcinomas stage III: a proposal for subdivision of Stage III. Cancer 2001;92:2754-2759.

29 Jass JR. Lymphocytic infiltration and survival in rectal cancer. J Clin Pathol 1986;39:585-589.

30 Yamazawa K, Seki K, Matsui H, et al. Significance of perivascular lymphocytic infiltrates in endometrial carcinoma. Cancer 2001;91:1777-1784.

31 Drayton DL, Liao S, Mounzer RH, et al. Lymphoid organ development: from ontogeny to neogenesis. Nat Immunol 2006;7:344-353.

32 Koukourakis MI, Giatromanolaki A, Sivridis E, et al. Inclusion of vasculature-related variables in the Dukes Staging System of colon cancer. Clin Cancer Res 2005;11:8653-8660.

33 Sternberg A, Sibirsky O, Cohen D, et al. Validation of a new classification system for curatively resected colorectal adenocarcinoma. Cancer 1999;86:782-792.

34 Sternberg A, Sibirsky O, Cohen D, et al. New approach to the substaging of node-positive colorectal adenocarcinoma. Ann Surg Oncol 1999;6:161-165.

35 Minsky BD, Cohen AM. Blood vessel invasion in colorectal cancer-an alternative to TNM staging? Ann Surg Oncol 1999;6:129-130.

36 Minsky B, Mies C. The clinical significance of vascular invasion in colorectal cancer. Dis Colon Rectum 1989;32:794-803.

37 Roberts S, Jonasson OL, McGrath R, et al. Clinical significance of cancer cells in the circulating blood: two- to five-year survival. Ann Surg 1961;154:362-371. 